\title{
Routine versus selective cardiac magnetic resonance in non-ischemic heart failure - OUTSMART-HF: study protocol for a randomized controlled trial (IMAGE-HF (heart failure) project 1-B)
}

Ian Paterson ${ }^{1 *}$, George A Wells ${ }^{2,3}$, Justin A Ezekowitz ${ }^{1}$, James A White ${ }^{5}$, Matthias G Friedrich ${ }^{6}$, Lisa M Mielniczuk ${ }^{2,3}$, Eileen O'Meara ${ }^{6}$, Benjamin Chow ${ }^{2,3,4}$, Rob A deKemp ${ }^{2,3}$, Ran Klein ${ }^{2,3}$, Carole Dennie ${ }^{2,3,4}$, Alexander Dick ${ }^{2,3,4}$, Doug Coyle ${ }^{2,3}$, Girish Dwivedi ${ }^{2}$, Miroslaw Rajda ${ }^{7}$, Graham A Wright ${ }^{8}$, Mika Laine ${ }^{9}$, Helena Hanninen ${ }^{9}$, Eric Larose ${ }^{10}$, Kim A Connelly ${ }^{14}$, Howard Leong-Poi ${ }^{14}$, Andrew G Howarth ${ }^{11}$, Ross A Davies ${ }^{2,3,4}$, Lloyd Duchesne, ${ }^{2,3}$, Seppo Yla-Herttuala ${ }^{15}$, Antti Saraste ${ }^{12}$, Paul Farand ${ }^{13}$, Linda Garrard ${ }^{2,3}$, Jean-Claude Tardif ${ }^{6}$, Malcolm Arnold ${ }^{5}$, Juhani Knuuti ${ }^{12,13,14,15}$, Rob Beanlands $2,3,4$ and Kwan L Chan 2,3,4

\begin{abstract}
Background: Imaging has become a routine part of heart failure (HF) investigation. Echocardiography is a first-line test in HF given its availability and it provides valuable diagnostic and prognostic information. Cardiac magnetic resonance (CMR) is an emerging clinical tool in the management of patients with non-ischemic heart failure. Current ACC/AHA/CCS/ESC guidelines advocate its role in the detection of a variety of cardiomyopathies but there is a paucity of high quality evidence to support these recommendations.

The primary objective of this study is to compare the diagnostic yield of routine cardiac magnetic resonance versus standard care (that is, echocardiography with only selective use of CMR) in patients with non-ischemic heart failure. The primary hypothesisis that the routine use of CMR will lead to a more specific diagnostic characterization of the underlying etiology of non-ischemic heart failure. This will lead to a reduction in the non-specific diagnoses of idiopathic dilated cardiomyopathy and HF with preserved ejection fraction.

Design: Tertiary care sites in Canada and Finland, with dedicated HF and CMR programs, will randomize consecutive patients with new or deteriorating HF to routine CMR or selective CMR. All patients will undergo a standard clinical echocardiogram and the interpreter will assign the most likely HF etiology. Those undergoing CMR will also have a standard examination and will be assigned a HF etiology based upon the findings. The treating physician's impression about non-ischemic HF etiology will be collected following all baseline testing (including echo \pm CMR). Patients will be followed annually for 4 years to ascertain clinical outcomes, quality of life and cost. The expected outcome is that the routine CMR arm will have a significantly higher rate of infiltrative, inflammatory, hypertrophic, ischemic and 'other' cardiomyopathy than the selective CMR group.

\footnotetext{
*Correspondence: ip3@ualberta.ca

'Mazankowski Alberta Heart Institute, University of Alberta, Edmonton, $A B$,

Canada

Full list of author information is available at the end of the article
} 


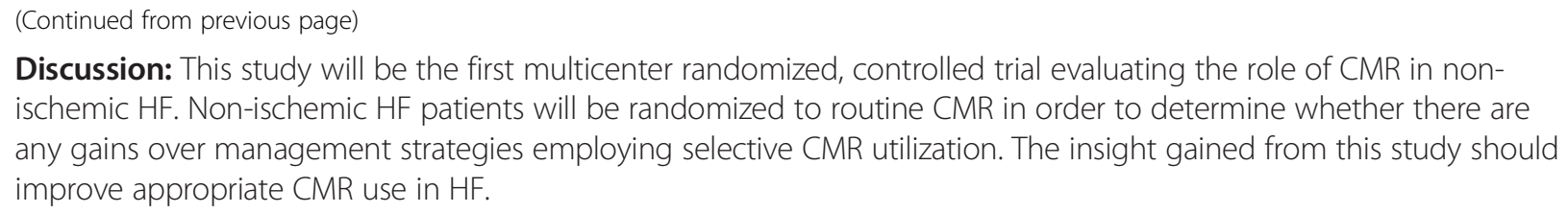

Trial registration: NCT01281384.

Keywords: Heart failure, Echocardiography, Cardiac magnetic resonance, Randomized controlled trial

\section{Background}

Innovations in imaging create the potential for earlier, less invasive and more accurate diagnoses of disease and may ultimately improve health outcomes. However, imaging is also one of the fastest growing health care expenditures. The 2005, the US Medicare Payment Advisory Committee found that the growth of cardiac imaging was nearly double any other cardiac procedure [1]. This underscores the need to ensure efficient development, implementation and use of this health care resource in order to provide value to patients and society. However an evaluation of the clinical and economic impact of cardiac imaging has lagged behind the pace of the technology. A recent review of current ACC/AHA guidelines found that only $11 \%$ of recommendations provide level of evidence A (multiple randomized controlled trials and meta analyses) and $48 \%$ have level of evidence $C$ (consensus opinion) [2]. In cardiac diagnostics, level of evidence $\mathrm{A}$ is found in only $2 \%$, level of evidence $\mathrm{C}$ in $17 \%$ and in $44 \%$ of recommendations there is no evidence provided. These observations underscore the need for more evidence based trials, particularly in cardiac imaging.

In 2007, the Canadian Institutes of Health Research New Frontiers Program consensus conference identified the urgent need for improved evaluation of imaging in relation to outcomes and translation from bench to bedside, particularly in heart failure (HF). In response, we established IMAGE-HF, a synergistic multi-disciplinary, multi-modality, multi-center Canada-Finland team comprised of seven Canadian sites and three Finnish sites. The main goal of IMAGE-HF is to evaluate cardiac imaging using existing clinical practice strategies for HF and their links to relevant outcomes (level 1 projects) and to create a unique translational platform for the evaluation of novel imaging biomarkers in animal models of HF and in HF patient populations (level 2 and 3 projects). IMAGE-HF 1-B, rOUTine versus Selective MAgnetic Resonance in non-ischemic HearT Failure-HF (OUTSMART-HF), will study the role of cardiac magnetic resonance (CMR) in non-ischemic heart failure.

\section{Statement of the problem}

Population studies reveal that $50 \%$ of HF patients have preserved ejection fraction (HFPEF) and a dismal prognosis similar to those HF patients with reduced ejection fraction (HFREF) [3-5]. Among the HFREF patients, 50\% have no significant underlying coronary artery disease [6]. The most common identifiable causes for non-ischemic HF include hypertension, valvular heart disease, arrhythmia, post-viral and acquired or inherited cardiomyopathies. However, standard measures of cardiac morphology and function obtained on imaging testing provide only limited information about HF etiology or prognosis [7] and, other than ejection fraction (EF), have a limited role in therapeutic decision-making.

Echocardiography is well suited for the assessment of HF patients because it is widely available and provides reliable information on cardiac performance in normal and diseased states. It is also considered the best imaging modality for valvular disease, a significant finding in 35\% of patients hospitalized with HF [8]. More recent technological advances such as tissue velocity and strain have shed new insights into pathophysiological mechanisms that may be particularly pertinent in HFPEF [9-11]. Furthermore, diastolic dysfunction determined by Doppler echocardiography is common in HF patients and has been shown to be a predictor of increased mortality during longitudinal follow-up [12]. Data from the Rochester Epidemiologic Program revealed that there was a lower risk of death in HF patients who had undergone echocardiograms compared to those who had not, suggesting that echocardiography improved care and should be part of the routine investigation of HF patients [13]. Given the available evidence and its extensive clinical experience, echocardiography is recommended as a class 1 indication in the initial investigation of all HF patients [14-16].

CMR is emerging as a valuable tool in the diagnosis of non-ischemic heart failure and is considered preferable or at least equivalent to other diagnostic tests in several cardiomyopathies [17]. Ventricular volumes and function derived from CMR is more accurate than standard echocardiography due to high inter-test and inter-observer reproducibility [18]. Moreover, CMR offers insight into myocardial tissue characteristics [19], with specific regional patterns of myocardial injury [20]. Non-infarct and infarct patterns have been reported in $28 \%$ and $13 \%$, respectively, of patients with HF and unobstructed coronary arteries [19]. In a recent single site study of 120 
patients with new onset HF, CMR had a 97\% accuracy for differentiating an ischemic from a non-ischemic etiology [21]. CMR has been used to characterize myocarditis [22-24], sarcoidosis [25], hypertrophic cardiomyopathy [26], hemochromatosis [27] and amyloidosis [28]. CMR thus offers a new class of diagnostic and prognostic parameters for personalized therapy and prevention of HF. However, there is a need to understand how such data impacts diagnosis, clinical decision-making and patient outcomes.

The diagnosis of HFPEF remains challenging as discussed in negative drug trials to date [29-31]. Diagnostic criteria incorporating clinical signs and symptoms, biomarkers, imaging and/or hemodynamic parameters have been proposed but not studied well [32]. Diffuse myocardial fibrosis as assessed by CMR has been utilized as a novel diagnostic target in HF [33] and cardiomyopathy subjects [34]. Patients with HFPEF are known to have increased myocardial collagen and fibrosis and hence CMR could be used to study this group.

Despite growing evidence of the utility for CMR in various cardiomyopathies, a comprehensive study examining its role in HF is lacking.

\section{Objectives}

\section{Primary objective}

To compare the diagnostic yield of routine cardiac magnetic resonance versus standard care (that is, echocardiography with only selective use of CMR) in patients with non-ischemic heart failure. The diagnostic categories of $\mathrm{HF}$ to be considered in this study include: idiopathic dilated cardiomyopathy, infiltrative cardiomyopathy, inflammatory cardiomyopathy, hypertrophic cardiomyopathy, heart failure with preserved ejection fraction (HFPEF), ischemic cardiomyopathy, mixed etiology and 'other' (for example, pericardial disease, adult congenital heart disease, arrhythmogenic right ventricular cardiomyopathy).

\section{Secondary objectives}

To determine the effects that routine use of CMR in non-ischemic HF has on therapeutic decisions on the Composite Clinical Endpoint (CCE), cardiac function, symptoms, quality of life (QoL), and costs. Ancillary measurements will include the safety of imaging tests and adverse reactions to gadolinium contrast agent.

\section{Hypotheses}

\section{Primary hypothesis}

Routine use of CMR (versus selective use) will lead to a more specific diagnostic characterization of the underlying etiology of non-ischemic heart failure. This will lead to a reduction in the non-specific diagnoses of idiopathic dilated cardiomyopathy and HFPEF.

\section{Secondary hypotheses}

Routine use of CMR will have a significant impact on treatment decisions, and will (1) lead to more disease specific therapies and/or (2) cause a significant change in the number and class of HF medications, during follow-up. The routine CMR group will also have improved clinical outcomes (CCE), symptoms and QoL and decreased costs compared to the standard of care group during follow-up.

\section{Design}

Randomized controlled trial comparing i) routine CMR to ii) echocardiography with selective CMR in patient with HF due to a non-ischemic cardiomyopathy (NICM) and/or HFPEF. Among patients enrolled in level 1 of IMAGE-HF, it is expected that 504 will have known NICM (or strongly suspected based on young age, absent risk factors and presenting history) and/or HFPEF (Figure 1).

Tertiary care sites (in Canada and Finland) with dedicated HF programs will participate in the study (Additional file 1). Consecutive patients will be enrolled at sites with CMR programs (defined as a minimum 200 cases/year) and randomized to routine CMR or selective CMR. Non-ischemic HF patients from sites with smaller CMR programs will be included in a registry of patients undergoing routine HF care (that is, selective use of CMR). All sites will receive approval from local ethics committees (UOHI REB protocol \# 2010422-01H) and patients will give written informed consent. Participants in the selective CMR arm may only undergo CMR when there is a suspicion or uncertainty for a diagnosis of: 1) infiltrative cardiomyopathy, 2) arrhythmogenic right ventricular cardiomyopathy, 3) adult congenital heart disease or 4) pericardial disease following standard HF care including echocardiography [35]. Other tertiary sites may be added in year two to three depending on recruitment needs and registry sites may become randomization sites if the experience and wait-time criteria are met.

\section{Study population}

We will recruit patients with either newly diagnosed HF within the past 12 months or established HF patients with deterioration/decompensation within the past 12 months.

\section{Inclusion criteria:}

Patients with new or worsening HF as above and:

1) age $>18$ years

2) working clinical diagnosis (known or highly suspected) of non-ischemic cardiomyopathy (NICM) or clinical diagnosis of HFPEF (signs or symptoms of heart failure with a LVEF $\geq 40 \%$ )

3) documented history of Class II-IV NYHA HF symptoms within the past 12 months 


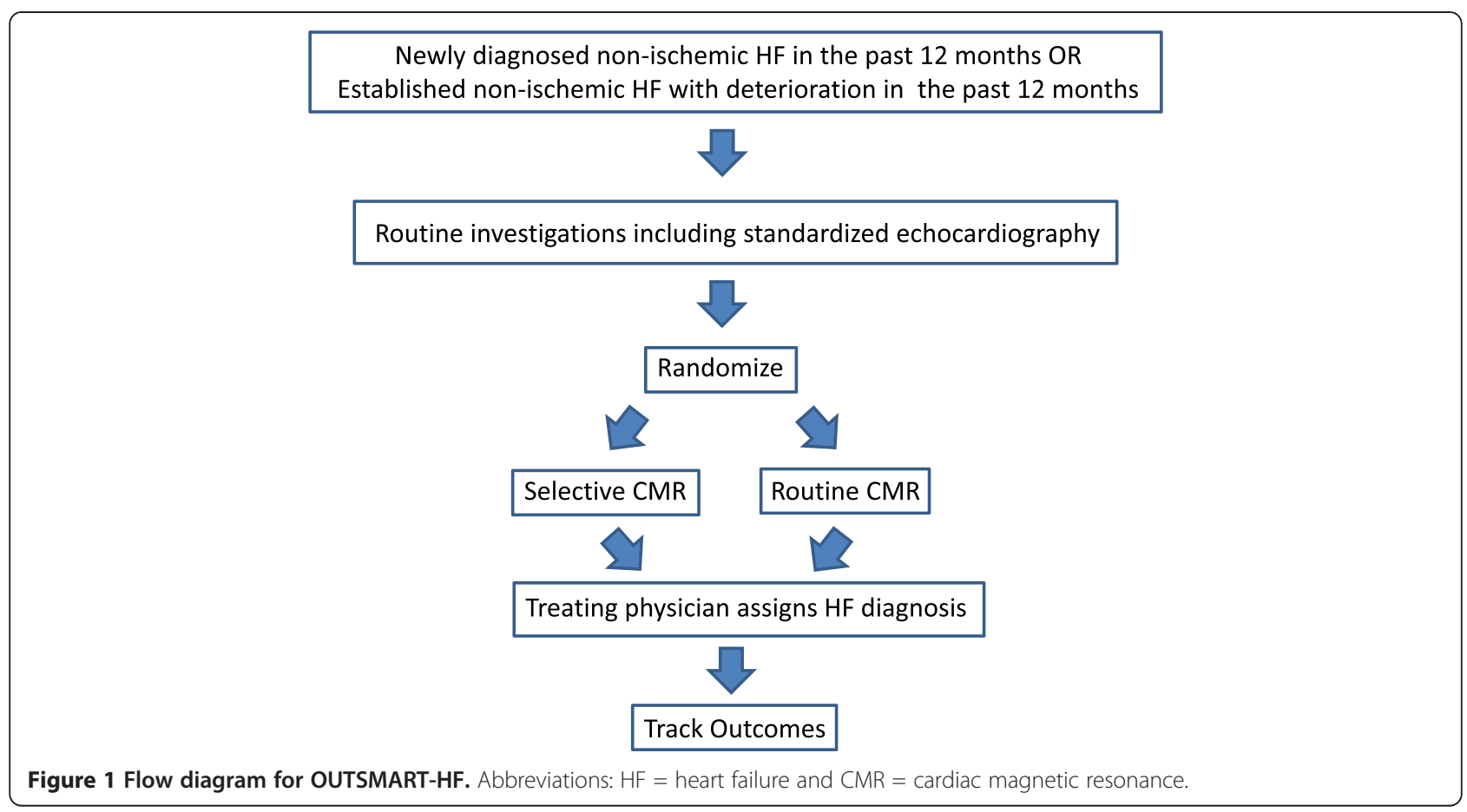

\section{Exclusion criteria:}

1) prior CMR and no major change in clinical condition

2) well-documented specific etiology (for example, known amyloidosis or hemochromatosis)

3) physician considers cause of heart failure is attributable to obstructive CAD

4) documented previous ST-segment elevation myocardial infarction (STEMI) (any territory) or non-ST-segment elevation myocardial infarction (NSTEMI) in the left anterior descending coronary artery territory

5) evidence of multi-vessel ischemia on stress imaging

6) ongoing need for advanced heart failure support (for example, inotropes or intra-aortic balloon pump)

7) severe valvular heart disease requiring surgery within the next six months

8) contraindications to CMR (for example, certain metallic implants, severe claustrophobia)

9) contraindications to gadolinium contrast agent (glomerular filtration rate $<30 \mathrm{ml} / \mathrm{min} / 1.72 \mathrm{~m}^{2}$, pregnancy)

10) severe medical conditions that significantly affect the patient's outcome (for example, active malignancy)

11) inability to give informed consent

\section{Description of interventions (randomized and registry participants)}

Baseline: demographic and clinical data will be collected from all participants on standardized case report forms. This data will be collected from the participant and the most recent, routine history and physical examination that have been completed by the treating physician. Quality of life questionnaires (Minnesota Living with Heart Failure (MLHFQ) and EuroQoL Quality of Life Scale (EQ5D)) will be administered. Patients at all sites will undergo standard HF investigations according to published Canadian guidelines [14,36,37]. Each center will have a HF lead, an echo lead and a CMR lead as well as a study co-ordinator.

\section{Echocardiography}

The performance and interpretation of echocardiography will be carried out according to established Canadian guidelines [38]. The echocardiography study and report must include the following measures: left ventricular (LV) size and systolic function (modified Simpson's biplane formula), LV diastolic function (E/e'), LV mass (Teichholz formula), left atrial (LA) volume (biplane formula), valvular function (Doppler), right ventricular function, pulmonary arterial pressures (if possible) and pericardial assessment. Appendix A. The feasibility of implementing these standards and collaboration among multiple centers has been demonstrated in prospective echocardiography trials such as the ASTRONOMER study [39]. The new 
components such as strain and tissue velocity will be obtained and measured as described [40]. A standardized case report form will be completed by the echocardiographer. The most likely HF etiology based upon the echocardiogram will be provided to the referring physician.

\section{CMR}

Following all HF investigations (including echocardiography), study participants randomized to routine CMR as well as those requiring selective CMR will undergo a conventional scan. The CMR protocol must include an assessment of biventricular volumes and systolic function, LV mass, LA volume (biplane formula), global and regional myocardial edema using $\mathrm{T} 2$ weighted sequences, and hyperemia and regional myocardial fibrosis using contrast enhanced T1 weighted sequences. Appendix A. CMR interpreters participating in this trial should report a minimum of 100 cases/year. Local CMR interpreters at each participating center will be expected to complete a standardized clinical report form. The most likely HF etiology based upon the CMR will be provided to the referring physician. All CMR data acquisition and reporting will conform to current published guidelines [41,42].

The echo and CMR reports will be integrated with the other clinical data when used by the treating physician to make a final clinical determination on the HF etiology.

\section{Primary outcome measure - frequency of definitive diagnoses}

Following the completion of all baseline testing (including echo \pm CMR), the treating physician will assign a diagnosis on a standardized template using all available information. The HF categories include: idiopathic dilated cardiomyopathy, infiltrative cardiomyopathy, inflammatory cardiomyopathy, hypertrophic cardiomyopathy, heart failure with preserved ejection fraction (HFPEF), ischemic cardiomyopathy, mixed etiology and other (for example, pericardial disease, adult congenital heart disease, arrhythmogenic right ventricular cardiomyopathy). The diagnosis of non-ischemic cardiomyopathies will be based upon the 2008 Canadian Cardiovascular Society Consensus conference guidelines [37].

\section{Expected result}

The routine CMR arm will have a significantly higher rate of infiltrative, inflammatory, hypertrophic, ischemic, mixed and other cardiomyopathies than the selective CMR group.

\section{Follow-up}

Clinical outcomes will be collected by telephone followup, and/or by hospital chart and electronic health record review at three and twelve months and then yearly up to four years, following completion of baseline scans. Data will be recorded on standardized case report forms. Quality of life questionnaires (MLHFQ and EQ5D) will be administered at each follow-up.

\section{Secondary outcome measures}

(i) Treatment effects: at three months and one year, the treating physician will be asked to reassess the HF etiology. The presence of each HF medication class will be re-assessed in addition to the overall number of cardiac medications. The presence of advanced HF therapies will additionally be recorded at each follow-up visit including: implantable device, electrophysiologic study/ablation, cardiac surgery/transplantation, and disease specific therapies (for example, phlebotomy for hemochromatosis; steroids for sarcoidosis).

(ii) Clinical endpoints: Composite Clinical Endpoints (CCE that is, death, cardiovascular (CV) death, HF admission), left ventricular function, QoL, referral to HF clinic, costs and safety will be assessed at three months, then annually for a minimum of one year and a maximum of four years.

Resource utilization and costs: regression methods will be used to assess the incremental costs associated with the routine use of CMR.

(iii) Echo/CMR variability: anonymized copies of CMR and echo studies will be sent to a core lab for interpretation in approximately $10 \%$ of cases in order to assess reproducibility and quality assurance of the results. Appendix A.

\section{Statistical analysis}

Descriptive statistics will be used to summarize the characteristics of the patients for each imaging technology on demographic, clinical and site related factors, and differences between these groups will be reviewed for their clinical significance.

\section{Analysis populations}

For the purposes of data analysis, three study populations will be considered: Intent-to-treat (ITT) population, As-treated population and Per-protocol population. The ITT population will be used for the main analysis for all primary and secondary objectives, except for the safety analysis where the As-treated population will be used. As a secondary analysis, the analyses will be repeated for the As-treated and Per-protocol populations. 


\section{Primary analysis}

For the primary analysis, the definitive diagnosis of cardiomyopathy between routine CMR versus echo with selective CMR, diagnosis will be compared using chisquare techniques. Potential confounding variables of the relationship between the imaging strategies and the primary endpoint will be assessed. In particular, propensity scores based on patient factors (for example, in/ outpatient, NYHA class, HF duration, diabetes, atrial fibrillation) and site factors (for example, time-toimaging, time-to-therapy) will be used in the analysis if necessary to adjust for potential differences. A logistic regression model will be used to assess the occurrence of the endpoints between the imaging strategies (model will include a group indicator variable) adjusting for any pertinent baseline differences identified.

\section{Secondary outcomes}

For the binary secondary outcomes, such as CCE, referral to HF clinic and HF therapies, chi-square tests will be used to compare the imaging strategies. Logistic regression analysis will be used for adjusting any pertinent baseline differences identified. For continuous secondary outcomes, such as LV function, MLHFQ and EQ5D, analysis of variance will be used to compare trends over time between the imaging strategies. Analysis of covariance will be used for adjusting any pertinent baseline differences identified. For assessing the reproducibility of echo/CMR results, reliability statistics, including intra-class correlations and kappas, will be calculated to compare CMR and echo studies with the core lab interpretations.

\section{Economic evaluation}

A cost minimization analysis comparing routine CMR versus standard care (echocardiography plus selective CMR) will be conducted. Analysis will be restricted to the follow-up period within the study database and will be conducted from the health care system perspective. Regression analysis will provide $95 \%$ confidence intervals around the estimate of incremental costs. In addition, univariate sensitivity analysis will be conducted to assess the robustness of the study's results to changing assumptions related to the unit costs of specific resource items.

\section{Safety analysis}

Safety will be evaluated by documenting all adverse events. Descriptive statistics (frequency distributions, numerical descriptors) and 95\% confidence intervals will be calculated. The As-treated population will be the main analysis population for this safety evaluation.

\section{Missing data}

'Missingness' is considered to be missing at random and mixed methods repeated measures (MMRM) and multiple imputation techniques will be used for handling missing data. In particular, for continuous outcomes at multiple time points MMRM will be used.

\section{Sample size justification}

Based on a study of patients with NICM (prior to availability of CMR) a myocardial biopsy adds a specific diagnosis to standard approaches in $15 \%$ of patients with NICM increasing the rate from $35 \%$ to $50 \%$ [7]. For the sample size determination, the estimated occurrence of a definitive diagnosis with CMR is $50 \%$ and echo (with selective use of CMR) is $35 \%$. For the primary analysis comparing routine CMR versus selective CMR with a sample size of 252 per group is required to detect a difference in the event rate of definitive diagnosis of cardiomyopathy of 35\% (selective CMR) versus 50\% (routine CMR), based on the Z-test with a pooled variance estimate, and after adjustment for $10 \%$ patient loss or withdrawal, we will be able to detect a difference in the event rate of definitive diagnosis of cardiomyopathy of 35\% (selective CMR) versus 50\% (routine CMR), a level of significance of 0.05 , power of $90 \%$ and an adjustment for $10 \%$ patient loss or withdrawal.

\section{Study management}

The IMAGE-HF trial is managed by an Executive Committee consisting of clinicians specialized in diagnostic imaging and/or heart failure and experts in biostatistics, physics and radiochemistry, as well as a larger Steering Committee consisting of members of the Executive Committee and representatives of all the initial study centers. In addition there is an events adjudication committee which will independently review and adjudicate each clinical event blinded to treatment randomization. Since all the imaging approaches are part of standard clinical practice, no interim analysis is planned but there will be an independent Data Safety Monitoring Board (DSMB) which will review the safety data on a periodic basis; the frequency of the meetings and the charter governing the DSMB will be finalized at the first meeting of the DSMB.

\section{Discussion}

Multicenter randomized controlled trials are needed to evaluate the emerging role of advanced cardiac imaging in heart failure. CMR is increasingly advocated as a useful tool to evaluate the diagnosis and prognosis of nonischemic HF patients, but to date there is little evidence to show that outcomes have improved. In IMAGE-HF 1-B, Canadian and Finnish tertiary care sites with access to CMR will randomize non-ischemic HF patients to routine CMR in order to determine whether there are any gains over current imaging strategies employing selective CMR utilization. All patients in this trial will undergo a 
comprehensive baseline echocardiogram as recommended in HF guidelines.

The design of this trial has several appealing aspects:

First, we will evaluate the role of CMR in HF in a real world setting. Given differences in MRI access, local imaging expertise and in the interpretation of guidelines, the use of CMR in non-ischemic HF can vary greatly in a tertiary care setting. In the proposed randomized trial, we will study whether routine CMR offer any diagnostic gains over a more judicious approach. Such a strategy will thus emulate centers with open CMR access versus those without and determine what if any impact this has on actual patient management. All imaging studies, echo and CMR, will be part of the overall clinical care received by the patient. We also feel that the multicenter design with varying level of CMR expertise and the inclusion of new or worsening HF patients will provide a real world experience.

Second, we will be able to determine whether CMR diagnoses in non-ischemic HF differ from echo diagnoses. The influence that the echo and CMR diagnoses have on the treating physician's HF diagnosis will be examined. Also, the reproducibility of the imaging diagnoses will be determined in 10\% of CMRs and echos as part of our quality assurance analysis. Third, the potential for routine CMR to guide HF pharmacotherapy and to initiate disease specific treatment will be evaluated. CMR should uncover HF etiologies, such as myocardial infiltration, that was not previously detected by echo and other baseline testing. The treating physician could then prescribe treatment that is more tailored to the underlying pathology. Fourth, we will determine the impact of routine CMR on the outcomes of non-ischemic HF patients. Most advanced cardiac imaging studies evaluate the diagnostic accuracy of a given modality with no assessment on patient outcome. In a secondary analysis, we will evaluate clinical outcomes including: a composite clinical end-point (death, CV death and HF admission) and two quality of life surveys: MLHFQ and EQ5D. We will also ascertain the feasibility of implementing a routine CMR approach in terms of safety and cost.

\section{Limitations}

Given that CMR provides insight into myocardial characterization, we expect that the etiology of heart failure will be better characterized in those HF patients undergoing routine CMR. However, in some instances additional investigations such as myocardial biopsy and invasive hemodynamics may be required to confirm CMR findings. Therefore, the final diagnosis of heart failure etiology may ultimately be determined by CMR driven ancillary testing. We will assess the impact of downstream resource utilization on diagnosis and outcome in the multivariable analysis as well as calculate the additional cost burden.

\section{Summary}

In largely single center studies, CMR has been shown to offer a diagnostic advantage in specific HF disease states however its role in undifferentiated non-ischemic HF is largely unknown. Thus IMAGE-HF is a clinically relevant and timely study in an era of growing health costs and increasing pressure for fiscal responsibility. Given the available evidence and local experiences, we are confident that routine CMR will offer diagnostic and therapeutic advantages that ultimately improve patient outcomes at affordable costs.

\section{Trial status}

Recruiting

\section{Appendix A}

Standardization and quality assurance (IMAGE-QA)

The IMAGE-HF QA program standardizes several important aspects of clinical imaging:

1. defining best current imaging practice for standardcare tests

2. disseminating advanced imaging technology and standards

3. promoting structured reporting and comprehensive imaging QA

4. ensuring consistent interpretation and patient management recommendations

For the IMAGE-HF project 1-B (OUTSMART-HF), this includes standard operating procedures (SOPs) for echocardiography (ECHO) and cardiac magnetic resonance (CMR), as well as structured reporting elements and quality assurance review by QA-CORE labs (SOPs and CRFs are posted on the IMAGE-HF website).

\section{Standard-care imaging protocols \\ Echocardiography (ECHO): \\ IMAGE-HF 1-AB SOP ECHO-etiology (MHI)}

Based on Montreal Heart Institute (MHI) CORE laboratory procedure manual for the IMAGE-HF 1-A study (AIMI-HF): TECHNICAL GUIDE FOR ECHOCARDIOGRAPHY October 2010 (E O'Meara, J-C Tardif). Detailed procedures are described for the required parasternal, apical and sub-costal view measurements, and instructions for transfer of DICOM format images to the MHI CORE lab.

\section{Advanced imaging protocols \\ Cardiac Magnetic Resonance (CMR):}




\section{IMAGE-HF $\quad$ 1-B/2-A SOP CMR-etiology-fibrosis (CanSCMR)}

Based on Canadian Society for Cardiovascular Magnetic Resonance (CanSCMR) protocol recommendations v1.3 April 2009. Imaging parameters are included for assessment of LV function, inflammation, fibrosis and mitral valve regurgitation.

\section{Common structured reporting elements}

ECHO:

\section{HF18C 1-B-etiology ECHO Report}

The following parameters are included on the interpretation CRF: LV structure, LV systolic and diastolic function parameters, LV segmental wall-motion scores, RV structure and systolic function, severity of valve stenosis and regurgitation. Clinical interpretation as selected from a list of the most likely echocardiographic diagnoses, and confirmation that the diagnosis was communicated to the referring physician, are captured on the CRF.

$C M R$ :

\section{HF19C 1-B-etiology CMR Report}

The following parameters are included on the interpretation CRF: LV and RV structure and systolic function parameters, LV myocardial edema and hyperemia, LV segmental wall-motion and tissue characterization scores, severity of valve regurgitation, and assessment of pericardial effusion. Clinical interpretation as selected from a list of the most likely CMR diagnoses, and confirmation that the diagnosis was communicated to the referring physician, are captured on the CRF.

\section{Quality assurance CORE lab reviews (QA-CORE)}

A subset of scans (10\%) are targeted for over-reading interpretation at an experienced site identified as the CORE lab for each imaging modality. The first two scans (and 5\% of the subsequent scans) from each imaging modality at each recruiting site are transferred to the corresponding modality QA-CORE lab for clinical interpretation and comparison to the site interpretation for quality assurance. Disagreements in the overall ECHO or CMR diagnosis are resolved by subsequent consensus review between the site and CORE lab, and recorded on the corresponding CRFs: HF18-QA, HF19-QA.

The QA-CORE lab for ECHO is established at the University of Ottawa Heart Institute, and the QA-CORE lab for CMR is at the University of Alberta.

\section{Additional file}

Additional file 1: Table S1. IMAGE-HF Participating Sites.

\section{Abbreviations}

CCE: Composite clinical endpoint; CMR: Cardiac magnetic resonance, CV: Cardiovascular; DSMB: Data and safety monitoring board; echo: Echocardiography; EF: Ejection fraction; HF: Heart failure; HFPEF: Heart failure with preserved ejection fraction; HFREF: Heart failure with reduced ejection fraction; ITT: Intent-to-treat; LA: Left atrial; LV: Left ventricular; MHFQ: Minnesota living with heart failure questionnaire; MMRM: Mixed methods repeated measures; NICM: Nonischemic cardiomyopathy; NSTEMI: Non-ST-segment elevation myocardial infarction; QoL: Quality of life; STEMI: ST-segment elevation myocardial infarction.

\section{Competing interests}

MF: serves on the board of directors and a shareholder of Circle CV Imaging Inc, Calgary, AB, Canada, a cardiovascular MR imaging software manufacturer, E O'M: research funding from Johnson \& Johnson for the cardiorenal-anemia syndrome in HF, BC: receives research and fellowship training support from GE Healthcare, research support from Pfizer and AstraZeneca, and educational support from TeraRecon Inc., R deK: consultant Jubilant DraxImage; research funding: Lantheus Medical Imaging, GE Healthcare and MDS Nordion; receives revenues from rubidium generator technology licensed to Jubilant Draxlmage: receives revenues from FlowQuant software sales, RK: consultant Jubilant DraxImage; receives revenues from rubidium generator technology licensed to Jubilant DraxImage; receives revenues from FlowQuant software sales, GW: support from GE Healthcare for MR work which is helping to cover costs of the scans on the research system, RB: consultant Lantheus Medical Imaging, DraxImage; research funding: Lantheus Medical Imaging, GE, MDS Nordion. All other authors declare no competing interests.

\section{Authors' contributions}

IP conceived the study, participated in its design and coordination, provided CMR quality assurance and drafted the manuscript. KLC helped conceive the study, participated in its design and coordination and provided echocardiography quality assurance. RB helped conceive the study and participated in its design and oversaw overall project coordination. GAW helped conceive the study and participated in its design and coordination as well as providing statistical support. JK helped conceive the study and participated in its design and coordinated operations in the Finnish sites. LM helped conceive the study and participated in its design and coordination. EO helped conceive the study and participated in its design and coordination. BC helped conceive the study and participated in its design and coordination. LG is involved in the study design and project management and helped to draft the manuscript. RdK and RK established and will monitor the standardization of the imaging modalities. DC designed and will coordinate the economic evaluation. JE contributed to design of trial and will be involved with conducting the trial. JAW contributed to design of trial and will be involved with conducting the trial. MF contributed to design of trial and will be involved with conducting the trial. CD contributed to design of trial and will be involved with conducting the trial. $A D$ contributed to design of trial and will be involved with conducting the trial. GD contributed to design of trial and will be involved with conducting the trial. MR contributed to design of trial and will be involved with conducting the trial. GW contributed to design of trial and will be involved with conducting the trial. ML contributed to design of trial and will be involved with conducting the trial. $\mathrm{HH}$ contributed to design of trial and will be involved with conducting the trial. EL contributed to design of trial and will be involved with conducting the trial. $\mathrm{KC}$ contributed to design of trial and will be involved with conducting the trial. HL-P contributed to design of trial and will be involved with conducting the trial. $\mathrm{AH}$ contributed to design of trial and will be involved with conducting the trial. RAD contributed to design of trial and will be involved with conducting the trial. LD contributed to design of trial and will be involved with conducting the trial. STY-H contributed to design of trial and will be involved with conducting the trial. AS contributed to design of trial and will be involved with conducting the trial. PF contributed to design of trial and will be involved with conducting the trial. JCT contributed to design of trial and will be involved with conducting the trial. MA contributed to design of trial and will be involved with conducting the trial. All authors read and approved the manuscript.

\section{Acknowledgements}

OUTSMART-HF is supported by the IMAGE-HF Team Grant funded by Canadian Institute of Health Research CIHR (team grant \# CIF 99470). lan Paterson is supported the Canadian Institutes of Heart Research, Alberta Innovates-Health Solutions and the Alberta Cancer Foundation. Rob Beanlands is a career investigator supported by the Heart and Stroke 
Foundation of Ontario and Tier 1 Research Chair supported by the University of Ottawa. EO is sponsored by les Fonds de Rercherche en Santé du Québec (Junior 2 researcher grant 2011 to 2015) for research on biomarkers and imaging in heart failure and chronic kidney disease. GD is supported by Banting Postdoctoral Fellowship (Canadian Institute of Health Research $(\mathrm{ClHR})$ ), Dowager Countess Eleanor Peel trust (Rothwell-Jackson Travelling Fellowship (United Kingdom)) and Whit and Heather Tucker Cardiology Endowed Fellowship (Ottawa, Canada). MF is sponsored by the Fonds de Rercherche en Santé du Québec, the Canadian Foundation for Innovation Leadership Funds and the Montreal Heart Institute Foundation.

\section{Author details}

${ }^{1}$ Mazankowski Alberta Heart Institute, University of Alberta, Edmonton, $A B$, Canada. ${ }^{2}$ Division of Cardiology, (including Cardiac Imaging, The National Cardiac PET Center, The Heart Failure Program, and the Cardiac Research Methods Center), Department of Medicine, University of Ottawa Heart Institute, Ottawa, ON, Canada. ${ }^{3}$ University of Ottawa, Ottawa, ON, Canada. ${ }^{4}$ Department of Radiology, The Ottawa Hospital, Ottawa, ON, Canada. ${ }^{5}$ London Health Sciences Center, London, ON, Canada. ${ }^{6}$ Montreal Heart Institute, Departments of Medicine and Radiology, Université de Montréal, Montreal, QC, Canada. 'Dalhousie University, Halifax, NS, Canada. ${ }^{8}$ Sunnybrook Health Sciences Center, Toronto, ON, Canada. ${ }^{9}$ Helsinki University Central Hospital, Helsinki, Finland. ${ }^{10}$ Université de Québec, Québec City, QC, Canada. ${ }^{11}$ University of Calgary, Calgary, AB, Canada. ${ }^{2}$ Turku PET Center, Turku, Finland. ${ }^{13}$ Université de Sherbrooke, Sherbrooke, QC, Canada. ${ }^{14}$ St. Michael's Hospital, Toronto, ON, Canada. ${ }^{15}$ Heart Center, Kuopio University Hospital, Kuopio, Finland.

Received: 11 March 2013 Accepted: 23 September 2013

Published: 12 October 2013

\section{References}

1. Shaw $L$, Narula J: Cardiovascular imaging quality-more than a pretty picture! JACC Cardiovasc Imaging 2008, 1:266-269.

2. Tricoci P, Allen JM, Kramer JM, Califf RM, Smith SC Jr: Scientific evidence underlying the ACC/AHA clinical practice guidelines. JAMA 2009, 301:831-841.

3. Owan TE, Hodge DO, Herges RM, Jacobsen SJ, Roger VL, Redfield MM: Trends in prevalence and outcome of heart failure with preserved ejection fraction. N Engl J Med 2006, 355:251-259.

4. Tribouilloy C, Rusinaru D, Mahjoub H, Souliere V, Levy F, Peltier M, Slama $M$, Massy Z: Prognosis of heart failure with preserved ejection fraction: a five year prospective population-based study. Eur Heart J 2008, 29:339-347.

5. Bhatia RS, Tu JV, Lee DS, Austin PC, Fang J, Haouzi A, Gong Y, Liu PP: Outcome of heart failure with preserved ejection fraction in a population-based study. N Engl I Med 2006, 355:260-269.

6. Horwich TB, MacLellan WR, Fonarow GC: Statin therapy is associated with improved survival in ischemic and non-ischemic heart failure. J Am Coll Cardiol 2004, 43:642-648.

7. Felker GM, Thompson RE, Hare JM, Hruban RH, Clemetson DE, Howard DL, Baughman KL, Kasper EK: Underlying causes and long-term survival in patients with initially unexplained cardiomyopathy. N Engl J Med 2000 342:1077-1084.

8. Stein GY, Kremer A, Shochat T, Bental T, Korenfeld R, Abramson E, Ben-Gal T, Sagie A, Fuchs S: The diversity of heart failure in a hospitalized population: the role of age. J Card Fail 2012, 18:645-653

9. Nagueh SF, Middleton K, Kopelen HA, Zoghbi WA, Quinones MA: Doppler tissue imaging: a noninvasive technique for evaluation of left ventricular relaxation and estimation of filling pressures. J Am Coll Cardiol 1997, 30:1527-1533.

10. Dokainish $H$, Sengupta R, Pillai M, Bobek J, Lakkis N: Assessment of left ventricular systolic function using echocardiography in patients with preserved ejection fraction and elevated diastolic pressures. Am J Cardiol 2008, 101:1766-1771.

11. Yip GW, Zhang Q, Xie JM, Liang YJ, Liu YM, Yan B, Lam YY, Yu CM: Resting global and regional left ventricular contractility in patients with heart failure and normal ejection fraction: insights from speckle-tracking echocardiography. Heart 2011, 97:287-294.
12. Bursi F, Weston SA, Redfield MM, Jacobsen SJ, Pakhomov S, Nkomo VT, Meverden RA, Roger VL: Systolic and diastolic heart failure in the community. JAMA 2006, 296:2209-2216.

13. Senni M, Rodeheffer RJ, Tribouilloy CM, Evans JM, Jacobsen SJ, Bailey KR, Redfield MM: Use of echocardiography in the management of congestive heart failure in the community. J Am Coll Cardiol 1999, 33:164-170

14. Arnold JM, Liu P, Demers C, Dorian P, Giannetti N, Haddad H, Heckman GA, Howlett JG, lgnaszewski A, Johnstone DE, Jong P, McKelvie RS, Moe GW, Parker JD, Rao V, Ross HJ, Sequeira EJ, Svendsen AM, Teo K, Tsuyuki RT, White M: Canadian Cardiovascular Society consensus conference recommendations on heart failure 2006: diagnosis and management. Can J Cardiol 2006, 22:23-45.

15. Lindenfeld J, Albert NM, Boehmer JP, Collins SP, Ezekowitz JA, Givertz MM, Katz SD, Klapholz M, Moser DK, Rogers JG, Starling RC, Stevenson WG, Tang WH, Teerlink JR, Walsh MN: HFSA 2010 comprehensive heart failure practice guideline. J Card Fail 2010, 16:e1-e194.

16. McMurray JJ, Adamopoulos S, Anker SD, Auricchio A, Bohm M, Dickstein K, Falk V, Filippatos G, Fonseca C, Gomez-Sanchez MA, Jaarsma T, Kober L, Lip GY, Maggioni AP, Parkhomenko A, Pieske BM, Popescu BA, Ronnevik PK, Rutten FH, Schwitter J, Seferovic P, Stepinska J, Trindade PT, Voors AA, Zannad F, Zeiher A: ESC guidelines for the diagnosis and treatment of acute and chronic heart failure 2012: the task force for the diagnosis and treatment of acute and chronic heart failure 2012 of the European society of cardiology. Developed in collaboration with the heart failure association (HFA) of the ESC. Eur Heart J 2012, 33:1787-1847.

17. Kramer CM, Budoff MJ, Fayad ZA, Ferrari VA, Goldman C, Lesser JR, Martin ET, Rajagopalan S, Reilly JP, Rodgers GP, Wechsler L, Creager MA, Holmes DR Jr, Merli G, Newby LK, Pina I, Rodgers GP, Weitz HH: ACCF/AHA 2007 clinical competence statement on vascular imaging with computed tomography and magnetic resonance. A report of the American College of Cardiology Foundation/American Heart Association/ American College of Physicians Task Force on Clinical Competence and Training. J Am Coll Cardiol 2007, 50:1097-1114.

18. Strohm O, Schulz-Menger J, Pilz B, Osterziel KJ, Dietz R, Friedrich MG Measurement of left ventricular dimensions and function in patients with dilated cardiomyopathy. J Magn Reson Imaging 2001, 13:367-371.

19. McCrohon JA, Moon JC, Prasad SK, MCKenna WJ, Lorenz CH, Coats AJ, Pennell $D J$ : Differentiation of heart failure related to dilated cardiomyopathy and coronary artery disease using gadolinium-enhanced cardiovascular magnetic resonance. Circulation 2003, 108:54-59.

20. Friedrich MG, Abdel-Aty H, Taylor A, Schulz-Menger J, Messroghli D, Dietz R: The salvaged area at risk in reperfused acute myocardial infarction as visualized by cardiovascular magnetic resonance. J Am Coll Cardiol 2008, 51:1581-1587.

21. Assomull RG, Shakespeare C, Kalra PR, Lloyd G, Gulati A, Strange J, Bradlow WM, Lyne J, Keegan J, Poole-Wilson P, Cowie MR, Pennell DJ, Prasad SK: Role of cardiovascular magnetic resonance as a gatekeeper to invasive coronary angiography in patients presenting with heart failure of unknown etiology. Circulation 2011, 124:1351-1360.

22. Abdel-Aty H, Boye P, Zagrosek A, Wassmuth R, Kumar A, Messroghli D, Bock P, Dietz R, Friedrich MG, Schulz-Menger J: Diagnostic performance of cardiovascular magnetic resonance in patients with suspected acute myocarditis: comparison of different approaches. J Am Coll Cardiol 2005, 45:1815-1822

23. Mahrholdt H, Goedecke C, Wagner A, Meinhardt G, Athanasiadis A, Vogelsberg $\mathrm{H}$, Fritz $\mathrm{P}$, Klingel K, Kandolf R, Sechtem U: Cardiovascular magnetic resonance assessment of human myocarditis: a comparison to histology and molecular pathology. Circulation 2004, 109:1250-1258.

24. Friedrich MG, Sechtem U, Schulz-Menger J, Holmvang G, Alakija P, Cooper LT, White JA, Abdel-Aty H, Gutberlet M, Prasad S, Aletras A, Laissy JP, Paterson I, Filipchuk NG, Kumar A, Pauschinger M, Liu P: Cardiovascular magnetic resonance in myocarditis: a JACC White Paper. J Am Coll Cardiol 2009, 53:1475-1487.

25. Smedema JP, Snoep G, van Kroonenburgh MP, van Geuns RJ, Dassen WR, Gorgels AP, Crijns HJ: Evaluation of the accuracy of gadolinium-enhanced cardiovascular magnetic resonance in the diagnosis of cardiac sarcoidosis. J Am Coll Cardiol 2005, 45:1683-1690.

26. Choudhury L, Mahrholdt H, Wagner A, Choi KM, Elliott MD, Klocke FJ, Bonow RO, Judd RM, Kim RJ: Myocardial scarring in asymptomatic or 
mildly symptomatic patients with hypertrophic cardiomyopathy. J Am Coll Cardiol 2002, 40:2156-2164.

27. Anderson $L$, Holden $S$, Davis B, Prescott E, Charrier CC, Bunce NH, Firmin DN, Wonke B, Porter J, Walker JM, Pennell DJ: Cardiovascular T2-star (T2*) magnetic resonance for the early diagnosis of myocardial iron overload. Eur Heart J 2001, 22:2171-2179.

28. Maceira AM, Joshi J, Prasad SK, Moon JC, Perugini E, Harding I, Sheppard MN, Poole-Wilson PA, Hawkins PN, Pennell DJ: Cardiovascular magnetic resonance in cardiac amyloidosis. Circulation 2005, 111:186-193.

29. Yusuf S, Pfeffer MA, Swedberg K, Granger CB, Held P, McMurray JJ, Michelson EL, Olofsson B, Ostergren J: Effects of candesartan in patients with chronic heart failure and preserved left-ventricular ejection fraction: the CHARM-Preserved Trial. Lancet 2003, 362:777-781.

30. Ahmed A, Rich MW, Fleg JL, Zile MR, Young JB, Kitzman DW, Love TE, Aronow WS, Adams KF Jr, Gheorghiade M: Effects of digoxin on morbidity and mortality in diastolic heart failure: the ancillary digitalis investigation group trial. Circulation 2006, 114:397-403.

31. Cleland JG, Tendera M, Adamus J, Freemantle N, Polonski L, Taylor J: The perindopril in elderly people with chronic heart failure (PEP-CHF) study. Eur Heart J 2006, 27:2338-2345.

32. Paulus WJ, Tschope C, Sanderson JE, Rusconi C, Flachskampf FA Rademakers FE, Marino P, Smiseth OA, De KG, Leite-Moreira AF, Borbely A, Edes I, Handoko ML, Heymans S, Pezzali N, Pieske B, Dickstein K, Fraser AG, Brutsaert DL: How to diagnose diastolic heart failure: a consensus statement on the diagnosis of heart failure with normal left ventricular ejection fraction by the Heart Failure and Echocardiography Associations of the European Society of Cardiology. Eur Heart J 2007, 28:2539-2550.

33. Iles L, Pfluger H, Phrommintikul A, Cherayath J, Aksit P, Gupta SN, Kaye DM, Taylor AJ: Evaluation of diffuse myocardial fibrosis in heart failure with cardiac magnetic resonance contrast-enhanced $\mathrm{T} 1$ mapping. J Am Coll Cardiol 2008, 52:1574-1580.

34. Dass S, Suttie JJ, Piechnik SK, Ferreira VM, Holloway CJ, Banerjee R, Mahmod M, Cochlin L, Karamitsos TD, Robson MD, Watkins H, Neubauer S: Myocardial tissue characterization using magnetic resonance noncontrast t1 mapping in hypertrophic and dilated cardiomyopathy. Circ Cardiovasc Imaging 2012, 5:726-733.

35. Hendel RC, Patel MR, Kramer CM, Poon M, Hendel RC, Carr JC, Gerstad NA, Gillam LD, Hodgson JM, Kim RJ, Kramer CM, Lesser JR, Martin ET, Messer JV, Redberg RF, Rubin GD, Rumsfeld JS, Taylor AJ, Weigold WG, Woodard PK Brindis RG, Hendel RC, Douglas PS, Peterson ED, Wolk MJ, Allen JM, Patel MR: ACCF/ACR/SCCT/SCMR/ASNC/NASCI/SCAI/SIR 2006 appropriateness criteria for cardiac computed tomography and cardiac magnetic resonance imaging: a report of the American College of Cardiology Foundation Quality Strategic Directions Committee Appropriateness Criteria Working Group, American College of Radiology, Society of Cardiovascular Computed Tomography, Society for Cardiovascular Magnetic Resonance, American Society of Nuclear Cardiology, North American Society for Cardiac Imaging, Society for Cardiovascular Angiography and Interventions, and Society of Interventional Radiology. J Am Coll Cardiol 2006, 48:1475-1497.

36. Arnold JM, Howlett JG, Dorian P, Ducharme A, Giannetti N, Haddad H, Heckman GA, Ignaszewski A, Isaac D, Jong P, Liu P, Mann E, McKelvie RS, Moe GW, Parker JD, Svendsen AM, Tsuyuki RT, O'Halloran K, Ross HJ, Rao V, Sequeira EJ, White M: Canadian Cardiovascular Society Consensus Conference recommendations on heart failure update 2007: prevention, management during intercurrent illness or acute decompensation, and use of biomarkers. Can J Cardiol 2007, 23:21-45.

37. Malcom J, Arnold O, Howlett JG, Ducharme A, Ezekowitz JA, Gardner MJ, Giannetti N, Haddad H, Heckman GA, Isaac D, Jong P, Liu P, Mann E, McKelvie RS, Moe GW, Svendsen AM, Tsuyuki RT, O'Halloran K, Ross HJ, Sequeira EJ, White M: Canadian Cardiovascular Society Consensus Conference guidelines on heart failure - 2008 update: best practices for the transition of care of heart failure patients, and the recognition, investigation and treatment of cardiomyopathies. Can J Cardiol 2008, 24:21-40.

38. Sanfilippo AJ, Bewick D, Chan KL, Cujec B, Dumesnil JG, Honos G, Munt B, Sasson Z, Tam J, Tomlinson C, Aboguddah A, Ahmed S, Ali M, Arsenault M, Ascah K, Ashton T, Baird M, Basmadjian A, Beique F, Blakeley M, Blais MJ, Burggraf G, Burwash I, Cochrane J, Fagan S, Giannoccaro P, Hughes W, Jones A, Jue J, Koilpillai C, et al: Guidelines for the provision of echocardiography in Canada: recommendations of a joint Canadian
Cardiovascular Society/Canadian Society of Echocardiography Consensus Panel. Can J Cardiol 2005, 21:763-780.

39. Chan KL, Teo K, Dumesnil JG, Ni A, Tam J: Effect of lipid lowering with rosuvastatin on progression of aortic stenosis: results of the aortic stenosis progression observation: measuring effects of rosuvastatin (ASTRONOMER) trial. Circulation 2010, 121:306-314.

40. Gorcsan J III, Tanaka H: Echocardiographic assessment of myocardial strain. J Am Coll Cardiol 2011, 58:1401-1413.

41. Hundley WG, Bluemke D, Bogaert JG, Friedrich MG, Higgins CB, Lawson MA, McConnell MV, Raman SV, van Rossum AC, Flamm S, Kramer CM, Nagel E, Neubauer S: Society for Cardiovascular Magnetic Resonance guidelines for reporting cardiovascular magnetic resonance examinations. J Cardiovasc Magn Reson 2009, 11:5.

42. Friedrich MG, Larose E, Patton D, Dick A, Merchant N, Paterson I: Canadian society for cardiovascular magnetic resonance (CanSCMR) recommendations for cardiovascular magnetic resonance image analysis and reporting. Can J Cardiol 2013, 29:260-265.

doi:10.1186/1745-6215-14-332

Cite this article as: Paterson et al:: Routine versus selective cardiac magnetic resonance in non-ischemic heart failure - OUTSMART-HF: study protocol for a randomized controlled trial (IMAGE-HF (heart failure) project 1-B). Trials $201314: 332$

\section{Submit your next manuscript to BioMed Central and take full advantage of:}

- Convenient online submission

- Thorough peer review

- No space constraints or color figure charges

- Immediate publication on acceptance

- Inclusion in PubMed, CAS, Scopus and Google Scholar

- Research which is freely available for redistribution 